\title{
ZGMTH $\begin{aligned} & \text { Zeitschrift der } \\ & \text { Gesellschaft für Musiktheorie }\end{aligned}$
}

14. Jahrgang 2017

Herausgegeben von

Ariane Jeßulat,

Ullrich Scheideler,

Kilian Sprau,

Christian Utz,

Verena Weidner,

Felix Wörner 


\section{ZGMTH}

Zeitschrift der Gesellschaft für Musiktheorie e.V.

https://doi.org/10.31751/zgmth

Wissenschaftlicher Beirat der Gesellschaft für Musiktheorie: Jean-Michel Bardez (Paris), Thomas Christensen (Chicago), Nicholas Cook (Cambridge), Jonathan Cross (Oxford), Hermann Danuser (Berlin), Helga de la MotteHaber (Berlin), Hartmut Fladt (Berlin), Allen Forte ( $t$, New Haven), Inga Mai Groote (Zürich), Renate Groth ( $t$, Bonn), Thomas Kabisch (Trossingen), Eckehard Kiem ( $t$, Freiburg), Clemens Kühn (Dresden), Nicolas Meeùs (Paris), Alexander Rehding (Cambridge, MA), Christian Martin Schmidt (Berlin), Michiel Schuijer (Amsterdam).

14. Jahrgang 2017

https://doi.org/10.31751/42 (Ausgabe 14/1); https://doi.org/10.31751/44 (Ausgabe 14/2)

Herausgeber:

Prof. Dr. Ariane Jeßulat, Alt-Friedrichsfelde 126, 10315 Berlin, AJessulat@aol.com

Dr. Ullrich Scheideler, Müllerstraße 150, 13353 Berlin, ullrich.scheideler@staff.hu-berlin.de

Dr. Kilian Sprau, Georg-Hann-Str. 17, 81247 München, kontakt@kiliansprau.de

Univ.-Prof. Dr. Christian Utz, Mariahilferstraße 56/27, A-1070 Wien, cu@christianutz.net

Dr. Verena Weidner, Wittstocker Str. 8, 10553 Berlin, verena.weidner@uni-erfurt.de

Dr. Felix Wörner, Manzentalstraße 37, 79541 Lörrach, felix.woerner@unibas.ch

Die Herausgeber sind per E-Mail erreichbar unter: redaktion@gmth.de.

Layout: Poli Quintana / Oliver Schwab-Felisch

Satz: Werner Eickhoff-Maschitzki, Umschlag: Oliver Schwab-Felisch

Notensatz und Grafik: Werner Eickhoff-Maschitzki

Erscheinungsweise: jährlich.

Beiträge und Anfragen senden Sie vorzugsweise in elektronischer Form an: redaktion@gmth.de.

Postzusendungen (z. B. Rezensionsexemplare von Druckschriften) nimmt entgegen:

Prof. Dr. Ariane Jeßulat, Alt-Friedrichsfelde 126, D-10315 Berlin.

Bezug über den Buchhandel oder direkt über Georg Olms Verlag, Hagentorwall 7, 31134 Hildesheim,

Tel.: +49(0)5121-15010, info@olms.de, www.olms.de.

Preise: Einzelband 44,- €, Abonnement 37,- € (zzgl. Versandspesen).

Für Mitglieder der Gesellschaft für Musiktheorie ist der Bezugspreis (exklusive Versand) durch den Mitgliedsbeitrag abgegolten.

Anzeigenannahme: Georg Olms Verlag.

Die Deutsche Bibliothek verzeichnet diese Publikation in der Deutschen Nationalbibliografie; detaillierte bibliografische Daten sind im Internet über https://www.deutsche-digitale-bibliothek.de abrufbar.

Das Werk ist urheberrechtlich geschützt. Jede Verwertung außerhalb der engen Grenzen des Urheberrechtsgesetzes ist ohne Zustimmung unzulässig. Das gilt insbesondere für Vervielfältigungen, Übersetzungen, Mikroverfilmungen sowie die Einspeicherung in und Verarbeitung durch elektronische Systeme.

(C) Georg Olms Verlag AG, Hildesheim 2018

Gedruckt auf säurefreiem und alterungsbeständigem Papier.

Alle Rechte vorbehalten.

Printed in Germany.

ISBN 978-3-487-15733-7

ISSN 1862-6742 


\section{Inhalt}

\section{JAHRGANG 2017, AUSGABE 1: ANALYSE UND AUFFÜHRUNG}

EDITORIAL

ARTIKEL

Thomas Glaser

Beethovens Violinkonzert als Modellfall

René Leibowitz' und Rudolf Kolischs Projekt einer

'werkgerechten Interpretation

Jan PhILIPP SpRICK

Form und Dramaturgie in Beethovens Violinkonzert

Zur Interpretation des Kopfsatzes durch Rudolf Kolisch

und René Leibowitz

TOBIAS BLEEK

"Das Geschriebene darf nicht ernst genommen werden das Geschriebene muß todernst genommen werden «

Zur Notation und Interpretation musikalischer Gesten

im Schaffen György Kurtágs

TOM Rojo Poller

The Interpretation is the Message

Komposition als angewandte Interpretation bei György Kurtág

Hubertus Dreyer, Pascal Horn

Schnittstellen zwischen performance und Analyse von Popmusik

Performative Produktionsprozesse in Pink Floyds Album

Wish You Were Here und Jordan Rudess' Coverversion von

Genesis' Dance on a Volcano

ROLAND HUSCHNER

Zur produktionsbezogenen Perspektive bei der Analyse von Popmusik

\section{REZENSIONEN}

Jan PhILIPP SPRICK

Steven Rings, Tonality and Transformation,

New York: Oxford University Press 2011 
FELIX WÖRNER

Stefan Keym (Hg.), Motivisch-thematische Arbeit als Inbegriff der Musik?

Zur Geschichte und Problematik eines sdeutschen Musikdiskurses

(Veröffentlichungen des Staatlichen Instituts für Musikforschung XXII:

Studien zur Geschichte der Musiktheorie 12),

Hildesheim: Olms 2015

KILIAN SPRAU

Johan van Beek, Klangrede am Klavier. Aufführungspraxis im 18. und 19. Jahrhundert, Kassel: Bärenreiter 2016 
EDITORIAL

ARTIKEL

MARTE AUER

"Wir wollen Gesetze aufspüren«

Anton Weberns verworfene Skizzen zu einem dritten Satz

der Symphonie op. 21

LÁsZLó VIKÁRIUS

Zur Bedeutung von Dokumenten kompositorischen und analytischen Denkens

Béla Bártoks Arbeit mit zyklischen Themen

VERA FUnK

Einen Anfang finden

György Ligetis Skizzen und Entwürfe zu den Drei Phantasien nach

Friedrich Hölderlin für 16-stimmigen gemischten Chor a cappella

KILIAN SPRAu

Breit über mein Haupt dein schwarzes Haar

Vier auktoriale Versionen von Richard Strauss' Schack-Vertonung

op. 19/2, betrachtet unter performativem Aspekt

Marc Neufeld

Eine Systematik diatonischer Skalen

\section{KLEINERE BEITRÄGE}

AdOLF NOWAK

Kritische Anmerkungen zu dem Aufsatz von Hermann Danuser:

"Apollinische Fundamente. Über Adolf Nowaks Buch Musikalische Logik»

ZGMTH 13/2 (2016), 355-375

\section{REZENSIONEN}

Michael Polth

Stefan Prey, Algorithmen zur Satztechnik und ihre Anwendung auf die Analyse, Phil. Diss., Universität Osnabrück 2012 Immanuel Ott, Methoden der Komposition bei Josquin Des Prez und seinen Zeigenossen (= Schriften der Musikhochschule Lübeck, Bd. 1), Hildesheim: Olms 2014 
HaRTMUt FLADT

Verena Weidner, Musikpädagogik und Musiktheorie. Systemtheoretische Beobachtungen einer problematischen Beziehung

(= Perspektiven musikpädagogischer Forschung, Bd. 3),

Münster: Waxmann 2015

UlLRICH SCHEIDELER

Thomas Ahrend / Matthias Schmidt (Hg.), Webern-Philologien

(= Webern-Studien. Beihefte der Anton Webern Gesamtausgabe, Bd. 3),

Wien: Lafite 2016 